\title{
A Mathematic Method to Adjust MLC Leaf End Position for Accuracy Dose Calculation in Carbon Ion Beam Radiation Therapy Treatment Planning System
}

\section{Yan-Shan Zhang Tumor Hospital \\ Yan-Cheng Ye Tumor Hospital \\ Jia-Ming Wu ( $\sim$ jiaming.wu@chmsc.com ) \\ Yee Zen General Hospital}

Heavy lon Center of Wuwei Cancer Hospital, Gansu Wuwei Academy of Medical Sciences, Gansu Wuwei

Heavy Ion Center of Wuwei Cancer Hospital, Gansu Wuwei Academy of Medical Sciences, Gansu Wuwei

\section{Research Article}

Keywords: multi-leaf collimator, leaf end position, offset correction, carbon ion beam

Posted Date: July 15th, 2021

DOI: https://doi.org/10.21203/rs.3.rs-707410/v1

License: (c) (i) This work is licensed under a Creative Commons Attribution 4.0 International License.

Read Full License 
A mathematic method to adjust MLC leaf end position for accuracy dose calculation in carbon ion beam radiation therapy treatment planning system

Yan-Shan Zhang ${ }^{1}$, Yan-Cheng Ye1, Jia-Ming $\mathrm{Wu}^{1,2,3^{*}}$

${ }^{1}$ Heavy Ion Center of Wuwei Cancer Hospital; Gansu Wuwei Academy of Medical Sciences;

Gansu Wuwei Tumor Hospital, Wuwei city, Gansu province, China.

2Department of Medical Physics, Chengde Medical University, Chengde City, Hebei

Province, China.

${ }^{3}$ Department of Radiation Oncology, Yee Zen General Hospital, Tao Yuan City, Taiwan

First authors (co-authors, equal contribution): Zhang Yan Shan ${ }^{1}$

${ }^{*}$ Corresponding authors (co-corresponding authors, equal contribution):

Yan-Cheng Ye', Jia-Ming $\mathrm{Wu}^{1,2,3^{*}}$

E-mail: jiaming.wu@chmsc.com 
Phone : 188-7358-8300

Fax : 0735-2343382

Running title: On axis MLC leaf end correction

Key words: multi-leaf collimator, leaf end position, offset correction, carbon ion beam

\section{Conflicts of Interest Notification :}

There are no actual or potential Conflicts of Interest in this study. This manuscript has not been published nor concurrently submitted for publication elsewhere. 


\section{ABSTRACT}

\section{Introduction:}

We present a mathematic method to adjust the leaf end position for dose calculation correction in carbon ion radiation therapy treatment planning system.

\section{Methods and Materials:}

A struggling range algorism of $400 \mathrm{MeV} / \mathrm{n}$ carbon ion beam in nine different multi-leaf collimator (MLC) materials was conducted to calculate the dose $50 \%$ point in order to derive the offset corrections in carbon ion treatment planning system (ciPlan). The visualized light field edge position in treatment planning system is denoted as $X_{\text {tang.p }}$ and MLC position $\left(X_{\text {mlc.p }}\right)$ is defined as the source to leaf end mid-point projection on axis for monitor unit calculation. The virtual source position of an energy at $400 \mathrm{MeV} / \mathrm{n}$ and struggling range in MLC at different field sizes were used to calculate the dose $50 \%$ position on axis. On-axis MLC offset (correction) could then be obtained from the position corresponding to $50 \%$ of the central axis dose minus the $X_{\text {mlc.p }}$ MLC position. 


\section{Results:}

The precise MLC position in carbon ion treatment planning system can be used an offset to do the correction. The offset correction of pure tungsten is the smallest among the others due to its shortest struggling range of carbon ion beam in MLC. The positions of 50\% dose of all MLC materials are always located in between $X_{\text {tang.p }}$ and $X_{\text {mlc.p }}$ under the largest field of $12 \mathrm{~cm}$ by $12 \mathrm{~cm}$.

\section{Conclusions:}

MLC offset should be adjusted carefully at different field size in treatment planning system especially of its small penumbra characteristic in carbon ion beam. It is necessary to find out the dose $50 \%$ position for adjusting MLC leaf edge on-axis location in the treatment planning system to reduce dose calculation error. 


\section{carbon ion beam radiation therapy treatment planning system}

\section{INTRODUCTION}

In most commercial photon radiation therapy facilities such as linear accelerators,

multi-leaf collimator $(\mathrm{MLC})$ systems are used to improve the dose profile of the geometry

penumbra and the transmission penumbra [1]. MLC not only used commonly as treatment

accessories in photon, but also adopted in heavy charged particles therapy such as

carbon ion beam treatment [2]. The coincidence between the 50\% dose position and the

light field of photon beam cannot be taken for granted with the non-divergent geometry

that is found in the curved-leaf linear type of collimator system, the $50 \%$ dose position

must be verified during MLC system acceptance [3]. Not like the photon, MLC systems

utilize designs with rounded leaf ends to improve the coincidence of the radiation $50 \%$

point with projected light field edge, the shape of MLC leaf end in charged particle is

rectangular [4]. The struggling range of carbon ion beam in MLC saves troubles with the rounded leaf end design caused by photon attenuation in MLC [5]. One of the most 
important principles of MLC design is to reduce the differences between the dose $50 \%$

points and the projected light field edge on axis [6]. The characteristic of the projected light field edge locations and the definition of MLC position as well as the dose $50 \%$

points in photon treatment planning system need to be corrected before patients' treatment monitor units are calculated. The MLC position in planning and its relative radiation dose $50 \%$ point of the carbon ion MLC are also needed to be corrected and implemented in the computerized treatment planning system for accuracy monitor unit calculation [7]. In this work, we illustrate the specific issues to carry out dose calculation of a rectangular end MLC system with an offset correction in carbon ion beam.

\section{Materials and Methods}

This work presented here was performed with $400 \mathrm{MeV} / \mathrm{n}$ on a carbon ion therapy facility established by the Institute of Modern Physics (IMP), China. The IMP affiliated with the Chinese Academy of Sciences (CAS), was founded in 1957 in Lanzhou, China. In order to take the advantage of full usage of the research facilities at IMP, the National 
Laboratory of Heavy Ion Accelerator, Lanzhou (NLHIAL) was established at IMP in 1991 [8].

Our WuWei Heavy lon Center, Wuwei Cancer $\underline{H}$ ospital, GanSu, China (WHICH) heavy ion facility established by IMP, CAS at 2014, was the first generation commercialized product transformed from laboratory-based cancer treatment facility in China. Our facility was a modification from the prototype of Heavy lon Research Facility in Lanzhou (HIRFL) and started to treat patients in earlier 2020. Our WHICH consists an ECR ion source, an injection cyclotron SFC (energy constant $\mathrm{K}=69$ ), a cyclotron SSC (energy constant $\mathrm{K}=$ 450) as an injector offering charged particles to the main synchrotron ring to accelerate sufficient particle energy and flux for four treatment rooms use-room 1, horizontal nozzle alone with scanning beam; room 2(for clinical use only), vertical+ horizontal nozzles of passive scatter beam; room 3, vertical nozzle alone with scanning beam; room 4, $45^{\circ}$ nozzle alone with passive scatter beam for cancer patients treatment. At WHICH, our home-made ciPlan treatment planning system was used for carbon ion dose calculations.

Room 1, 3, 4 are not ready for clinical services. Dose profiles of MLC fields were measured for room 2 passive scatter beam and implemented to the ciPlan for dose calculation in 
this study.

Nine MLC materials including struggling range listed at table 1 were adopted for this study.

The subscript denotes the percentage compositions of each MLC materials. According to IMP previous Monte Carlo simulation, the platform was v8.2/GEANT4-10-05-patch-01 with QGSP_BERT_HP_EMY package of Gate (GEANT4 Application for Tomographic Emission) [9]. The geometric dimensions of $\mathrm{WHICH}$ are showed in figure 1. All on-axis profiles were measured with a certain visual light field (nominal light field) at a SAD of $263.3 \mathrm{~cm}$ to determine the point receiving $50 \%$ of the central axis dose. The projection of the nominal light field at SAD $263.3 \mathrm{~cm}$ was adopted as a setup condition for dose profile measurements in water phantom, but the geometry of the tangential interaction on $\mathrm{x}$ axis $\left(X_{\text {tang, }}\right)$ was derived from $X_{\text {mlc,p }}$ (planning system defined leaf position) in ciPlan treatment planning system; furthermore, the corresponding dose $50 \%$ point to the central axis dose of $X_{m i c, p}$ was calculated by mathematical methods in this study. Once dose $50 \%$ point was decided, the on-axis correction "offset" could be obtained by subtraction of the point corresponding to $50 \%$ of the central axis dose from the position of $X_{\text {mlc.p. }}$. 


\section{II.A. Geometry specifications}

\section{A.1 Nominal light field}

Nominal light field means the size of the visualized light field that is set for patient

treatment and for dose profile measurements.

\section{A.2 $X_{\text {tang.p }}$-the position of light field projection edge interaction on axis}

According to Fig. 2, the bottom of carbon ion MLC rectangular leaf end determines $X_{\text {tang.p, }}$ which is the intersection of a prolonged line from the source to point $j$ with the isocentre horizontal axis at a SAD of $263.3 \mathrm{~cm}$. $X_{\text {tang.p }}$ is used quantitatively to describe the leaf edge in treatment planning systm, while the nominal light field (visualized light field) edge is used qualitatively by humans to check the boundary of the treatment area.

\section{A.3 $\mathrm{X}_{\mathrm{mlc} . \mathrm{p}}-$ definition of leaf position in treatment planning}

$X_{m l c p}$ is the intersection of a line from the source to the leaf tip ( $m$ in Fig. 2) with SAD 263.3 $\mathrm{cm}$ on axis. Patient dose calculations are based on this point in the treatment planning 
system.

\section{A.4. Direction of the MLC}

When the MLC travels away from the central axis (the field size becomes larger), the direction is denoted as positive (" $+"$ in all figures). When the MLC travels closer to or crosses over the central axis, the direction is denoted as negative (" $-"$ in all figures).

\section{A.5. Virtual source position of $400 \mathrm{MeV} / \mathrm{n}$ carbon ion beam}

A pencil carbon ion beam is spread into a broader beam after passing through the primary collimator, beam monitor, scatterer, ridge filter, ridge shifter and the range shifter that appears to diverge from a point _this point is so called the virtual source. The virtual source position may be defined as an intersection point of the back-projections along the most probable directions of carbon ions motion at the patient surface. Field size magnification of the $50 \%$ width of the beam profiles on Gaf chromic film with different distance was used for determining the virtual source position of carbon ion beam.

The virtual source position f was measured by the definition bellowed: 
$\frac{F S_{S A D, f}}{F S_{f+g}}=\frac{f}{f+g}$

$f=\frac{g}{\frac{F S_{f+g}}{F S_{S A D, f}}-1}$

Where $F S_{S A D, f}$ denotes the field size at SAD $263.3 \mathrm{~cm}$. The maximum field size of our WHICH carbon ion beams is $12 \mathrm{~cm} \times 12 \mathrm{~cm}$ at the isocenter of $263.3 \mathrm{~cm}$. A field size of $8 \mathrm{~cm}$ x $8 \mathrm{~cm}$ with gaps upstream or downstream were adopted for the virtual source position measurement in this study.

$F S_{f+g}$ denotes the field size at SAD $263.3 \mathrm{~cm}$ with gaps upstream or downstream, here we adopted the upstream and downstream with a gap of $-15 \mathrm{~cm}$ (close to source) and +15

cm (away from source), respectively.

$\mathrm{f}$ is the virtual source position and is the intersection point of the back-projections along the most probable directions of carbon ions motion at measurement devices surface.

A.6. GAF chromic film for measuring the virtual source position of $400 \mathrm{MeV} / \mathrm{n}$ carbon ion beam 
We used Gaf Chromic EBT3 films (Ashland Specialty Ingredients GP, NJ USA; Lot \# 04022001,

Exp. Date: April 2021) for determining the virtual source position of $400 \mathrm{MeV} / \mathrm{n}$ carbon ion

beam in this study. The film processing and dose profile measurements followed the

international protocols [10]. A pre-exposure technique was used for the calibration curve

derivation [11]. This was performed by giving each film a priming dose of 2 Gy to homogenize the

film density using our WHICH facility with a dose of $1 \mathrm{~Gy}$ at carbon ion energy of $400 \mathrm{MeV} / \mathrm{u}$. We

then measured the dose homogeneity using a densitometer. Graded doses of 5, 10, 15, 40, 60, 80,

100, 150 and 200 cGy were given to the GAF chromic film to obtain the Hurter-Driffield calibration

curve (H-D curve).

All exposed films of depth dose curve were then scanned with an Epson Expression 11000XL

scanner in the 48-bit RGB mode (16 bits per color), and the data were saved as tagged image file

format (TIFF) and analyzed by the VariSoft imaging procession software. A red filter was placed on

top of the GAF films before scanning to increase the slope of the H-D curve, thereby raising the

resolution of the dose-OD curves [12 ].

The field size derived from dose $50 \%$ of the dose profile at isocenter was then compared to an 
upstream and downstream films with a gap of $-15 \mathrm{~cm}$ and $+15 \mathrm{~cm}$ for determining the virtual source position.

\section{A.7. The $50 \%$ dose position: $\mathrm{X}_{50 \%}$}

The radiation field size is defined as the lateral distance between the $50 \%$ isodose line $\left(X_{50 \%}\right)$ at a reference depth. In photon beam, the dose $50 \%$ of the central axis dose is determined by the attenuation of radiation in $\mathrm{MLC}$, while in carbon ion beam, the struggling range dominate the position of $\mathrm{X}_{50 \%}$. When the MLC moves near to or away

from the central axis (Fig. 2), the $X_{50 \%}$ position might locate at point $n$ (right to $X_{\text {mlc.p }}$ ) or point $k$ (left to $X_{m l c . p}$ ), respectively. This depends on the struggling range in MLC (denoted as $\overline{g f}$ or $\overline{b d}$ in Fig. 2).

\section{A.8. Determination of dose $50 \%$ by struggling range of carbon ion beam in MLC}

The number of beam nuclei that survive passage through the MLC, $\mathrm{N}$ can be determined from the total number of carbon ion particles interaction events in MLC. This particles number is compared with the total number of incident nuclei, $N_{B}$, as determined 
from the total number of events in the collision history [13].

$N=N_{B} e^{\frac{-x t}{\lambda t}}$

where $x_{t}$ is the thickness of tungsten and, $\lambda t$ is the interaction mean free path (MFP), in other word, struggling range in tungsten MLC.

$N=N_{B} e^{\frac{-x t}{\lambda t}}$

Let $\mathrm{N}=0.5$

$N_{B}=1$, then $0.5=e^{\frac{-x t}{\lambda t}}, x_{t}$ is the half value layer of a certain carbon ion energy in MLC material

For example, $\lambda t=27 \mathrm{~mm}$ for tungsten at a carbon ion energy of $400 \mathrm{MeV} / \mathrm{n}$, then

$\ln (0.5)=\frac{-d 50}{27 m m}$

$d_{50}=1.8711 \mathrm{~cm}$, which means the path length to reduce dose to $50 \%$ of a carbon energy $400 \mathrm{MeV} / \mathrm{n}$ in tungsten MLC is $1.8711 \mathrm{~cm}$. 
Figure 2 shows a schematic drawing of a mathematical model for deriving the on-axis $50 \%$ dose position $\left(X_{50}\right), X_{\text {tang,p }}$ and $X_{m l c, p}$ at an SAD of $263.3 \mathrm{~cm}$. In figure 2, the precise position of the light field edge $\left(X_{\text {tang,p }}\right)$ was transformed from $X_{m l c, p}$ (denoted as " $m$ " in this figure) which was defined as MLC position in treatment planning system.

Once the MLC position is confirmed, the dose $50 \%$ position can be derived by the procedure in Appendix A.

\section{A.9. Offset definition}

The patient treatment monitor unit calculation was based on $X_{m c_{c} p}$ in the treatment planning system. The definition of the adjustment offset is as follows: The offset is equal to the $50 \%$ dose position minus the position of $\mathrm{X}_{\mathrm{mlc}, \mathrm{p}}$.

\section{Results}

\section{III.A. Virtual source position of $400 \mathrm{MeV} / \mathrm{n}$ carbon ion beam}

The result of virtual source point by field size magnification on films obtained by the 
back-projection of the $50 \%$ width of the beam profiles at different distances was found to be $5.5 \mathrm{~cm}$ downstream from the scatterer position in figure 1. In other words, the virtual source position was $257.8 \mathrm{~cm}$ from the patient treatment isocenter and the distance from virtual source to the bottom of the MLC is $191.3 \mathrm{~cm}$.

\section{III.B. On-axis offset correction of tungsten MLC leaf end position in treatment planning}

system.

Patient treatment field size is determined by plan designer according to the lesions of a PTV in treatment planning system. $X_{\text {tang.p }}$ is used quantitatively to describe the visualized light field leaf edge, while $X_{\text {mlc.p }}$ is the intersection of a line from the source to the leaf tip with an angle of $\theta$ and $\theta^{\prime}$ in treatment planning system, respectively. The dose $50 \%$ position $\mathrm{X}_{50 \%}$ ( straggling range in MLC) of tungsten was derived by the angle $\alpha$ listed in table 2 once $X_{\text {tang.p }}$ and $X_{\text {mlc.p }}$ is determined. The offset corrections listed in table 2 are equal to the dose of $50 \%$ position $\left(X_{50 \%}\right)$ minus the position of $X_{m l c, p}$ (MLC plan position in planning system). Light-Radiation agreement and the penumbra defined as dose 
profile between $20 \%-80 \%$ were also listed in table 2 .

\section{III.C. Secondary radiation equivalent dose and offset correction of different MLC materials}

The results of offset correction calculated by the procedures described in Appendix

A of nine different MLC materials with different field sizes were listed in table 3. The

largest and smallest offset corrections at the largest field size were pure alumina and

pure tungsten, respectively. The secondary radiation equivalent dose (mainly

composed of prompt gamma ray and neutrons in $10^{-4} \mathrm{~Sv}$ ) simulated by IMP Monte Carlo

simulation of the interactions of $400 \mathrm{MeV} / \mathrm{n}$ carbon ion beam with nine different MLC

materials were also listed in table 3.

Figure 3 is the schematic demonstration of the nine different MLC materials offset

corrections. Pure alumina is obviously segregated by the others due to its low $z$

characteristic.

\section{DISCUSSION}

The struggling range as well as the thickness of MLC were increased when the 
percentage of copper compositions of tungsten are increased in table 1. From the weights and mechanical driven point of view, the optimal material of MLC is pure tungsten.

The virtual source position was derived by field size magnification on films obtained by the back-projection of the $50 \%$ width of the beam profiles at different distances of the in and out direction (penetrate vertically through the paper) instead of up and down direction (parallel to the MLC movement demonstrated on paper) in figure 1. It was because the uncertainty of field size magnification on films obtained by up and down direction was larger than in and out direction due to the facility MLC movement is at up and down direction showed in figure 1.

The on-axis offset (the $50 \%$ dose position minus the planned leaf position) is used for accurate monitor unit calculation. Figure 2 shows $X_{\text {tang,p, }} X_{m l c, p r}$ and the on-axis position receiving $50 \%$ of the central axis dose (point $k$ or $n$ ). In photon beams, when MLC leaf travels close to the central axis, owing to gain enough attenuation the $50 \%$ dose position must project outside $X_{m l c, p}$ ( right to $X_{m l c, p}$ ) on point $n$. As the MLC leaf travels away from 
the central axis, the $50 \%$ dose projection position move inside $X_{m l c, p}$ ( left to to $X_{m l c, p}$ ) to point $\mathrm{k}$ for less attenuation in figure 2. Not like photons, the carbon ion $\mathrm{X}_{50 \%}$ are always located in between $X_{\text {tang,pr }} X_{m l c, p}$ regardless the field size due to the struggling range is enough for $50 \%$ dose attenuation. This offset adjustment can be of importance in clinical situations of split fields to avoid calculating over-dosage or under-dosage at treatment.

The maximum field size of our institute carbon ion beam is $12 \mathrm{~cm} \times 12 \mathrm{~cm}$, the corresponding offset and light-radiation agreement of half field size of $6 \mathrm{~cm}$ in table 2 were $-0.3689 \mathrm{~mm}$ and $-0.59767 \mathrm{~mm}$, respectively. The minus sign means the $\mathrm{X}_{50 \%}$ located in-between $X_{\text {tang,p }}$ and $X_{m l c, p .}$. For photon beams, the design of rounded leaf end structure reduce the distance of $X_{50 \%}$ to $X_{\text {tang,p }}$ and $X_{m l c, p}$ while in carbon ion beams, the rectangular leaf end have the same effect with rounded leaf end due to the struggling range of heavy charged particle in MLC.

Figure 3 shows the alumina was not suitable for MLC due to its low z material. The offset correction was increased because of the composition of different metal of all kinds 
of alloys increased leading to the increasement of struggling ranges in MLC.

The difference of secondary radiation equivalent dose of tungsten and alumina was only

$1.5 \times 10^{-4} \mathrm{~S} v$ showed in table 3, consider the weights and movement flexibility, tungsten is

still the best choice for fabricating MLC.

\section{Conclusions}

In this study, we illustrate that the accumulated and planned radiation doses may

not always be in agreement for MLC treatment fields at a carbon ion beam treatment planning system unless the offset is carefully adjusted.

It is necessary to find out the dose $50 \%$ position for adjusting MLC leaf edge on-axis

location in the treatment planning system to reduce dose calculation error.

We should keep in mind that patient treatment monitor unit calculations at extremely

settings such as split field in carbon ion beam could result in significant uncorrectable under-dosage or over-dosage in treatment planning calculation. 
Acknowledgement:

The author appreciates Professor Zhang Yan Shan, and Professor Ye Yan Cheng for their great contribution to this study. Professor Ye Yan Cheng is juxtaposed with correspondence author (co-corresponding authors with equal contribution).

This work was supported by the Funding: Key R\&D plan of Science and Technology Program of Gansu Province, China. (19YF3FH001).

Competing interest

None 
Figures legendary

Figure1.

The physical dimensions of carbon ion facility at $\mathrm{WHICH}$.

Figure2.

The definition of MLC nominal light field (visualized light field) edge, $X_{\text {tang.p, }}$ the intersection of a line from the source to the leaf tip with an angle of $\theta^{\prime}, X_{m l c . p r}$ and the dose $50 \%$ position $\mathrm{X}_{50 \%}$ ( straggling range) of tungsten in treatment planning system. 
Figure3.

Schematic demonstrates the offset corrections of different material for MLC. The offset correction is increased because of the composition of different metal increased leading to the increasement of struggling ranges.

Tables

Table1.

Nine MLC materials including the percentage compositions of each MLC materials

denoted as subscript symbols with struggling range were listed for this study.

Table2.

The tungsten MLC leaf end offset correction of $400 \mathrm{MeV} / \mathrm{n}$ carbon ion beam at different fields.

Table3. 
The MLC leaf end offset correction and secondary radiation equivalent dose for different materials of $400 \mathrm{MeV} / \mathrm{n}$ carbon ion beam at different field sizes.

\section{References}

1. Topolnjak R and Van der Heide UA. An analytical approach for optimizing the leaf design of a multi-leaf collimator in a linear accelerator. Phys Med Biol. 2008;

53(11):3007-21.

2. Kamada T, Tsujii H, Blakely EA, Debus J, De Neve W, Durante M, et al. Carbon ion radiotherapy in Japan: an assessment of 20 years of clinical experience. Lancet Oncol. 2015;16:e93-e100.

3. JM Wu, TF Lee, CM Kuo, CJ Chen, KY Hsiao, SA Yeh, A light field-based method to adjust rounded leaf end MLC position for split shape dose calculation correction in a 
radiation therapy treatment planning system. J App Clinc Med Phys, 2012; 13( 6):

$3-18$.

4. Tsujii H, Mizoe JE, Kamada T, Baba M, Kato S, Kato H, et al. Overview of clinical experiences on carbon ion radiotherapy at NIRS. Radiother Oncol. 2004;73 Suppl 2:S41-S49.

5. Krämer M, Jäkel O, Haberer T, Kraft G, Schardt D, Weber U. Treatment planning for heavy ion radiotherapy: Physical beam model and dose optimization. Phys Med Biol. 2000; 45:3299-3317.

6. M Sakama, Kanai, Y Kase, K Yusa, et al Design of ridge filters for spread-out Bragg peaks with Monte Carlo simulation in carbon ion therapy. Phys Med Biol 2012; 57 : $6615-6633$.

7. C K Ying, WA Kamil, IL Shuaib, M Naruhiro. An Improved Monte Carlo (MC) Dose Simulation For Charged Particle Cancer Therapy, Adv Nucl Res and Ener Development AIP Conf. Proc. 1584, 2014:97-100 
8. Qiang Li, Biomedical research with heavy ions at the IMP accelerators. Advan. in Spac. Resea. 2007; 40(4), 455-460.

9. XF ZHANG, QN MENG, ZG DAl, WQ CHEN, XU LIU, Qiang LI. Study on the Secondary Particles Produced in Different Material Leaves of Multi-leaf Collimator under Carbon Ion Irradiation. Nuclear Physics Review, 2020; 37(2): 217-224.

10. SG Ju, YC Ahn, SJ Huh, LJ Yeo. Film dosimetry for intensity modulated radiation therapy: dosimetric evaluation. Med Phys. 2002; 29(3):351-55.

11. T Cheung, MJ Butson, Yu PKN. Experimental energy response verification of XR type T radiochromic film. Phys Med Biol. 2004; 49(21):371-76.

12. Cheung T, Butson M, Yu PKN. Post-irradiation colouration of Gafchromic EBT radiochromic film. Phys Med Biol. 2005; 50(20):281-85.

13. L. C. NORTIICLIFFE, R. F. SCHILLING RANGE, STOPPING - POWER TABLES FOR HEAVY IONS, NUCLEAR DATA TABLES, 1970; A7: 233-463. 
Figures

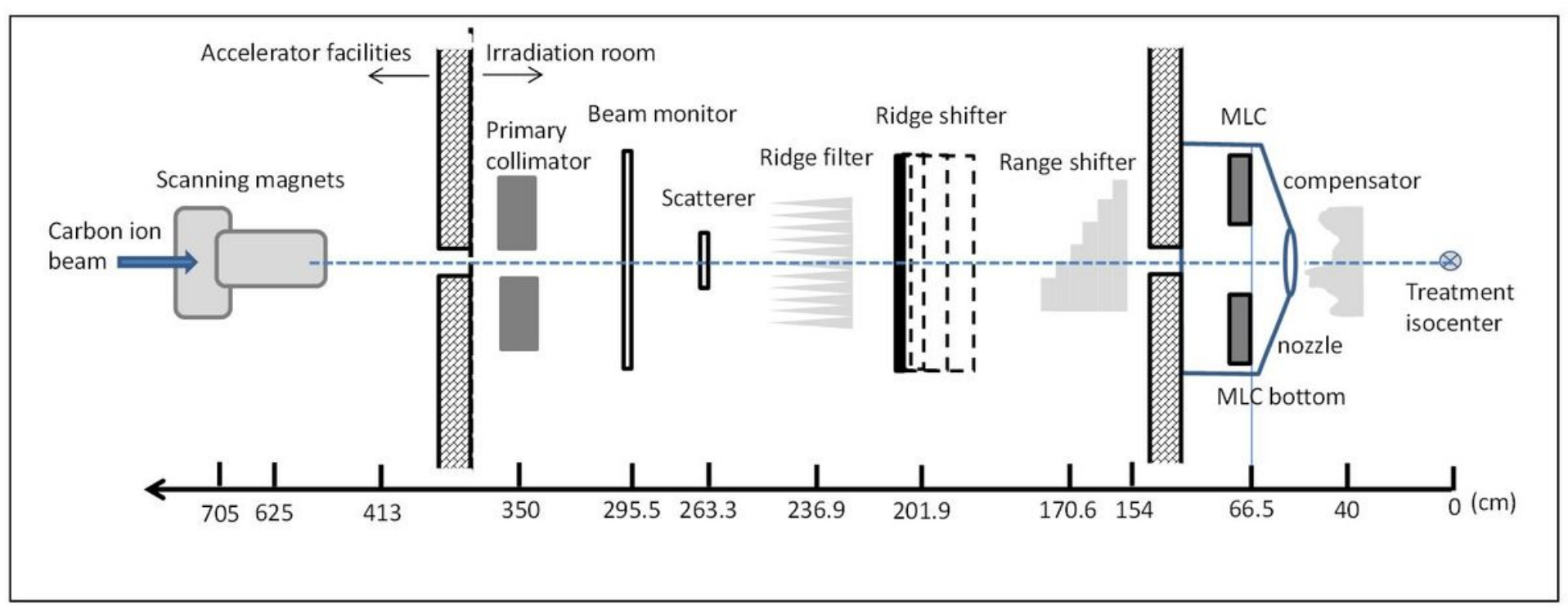

Figure 1

The physical dimensions of carbon ion facility at $\mathrm{WHICH}$. 


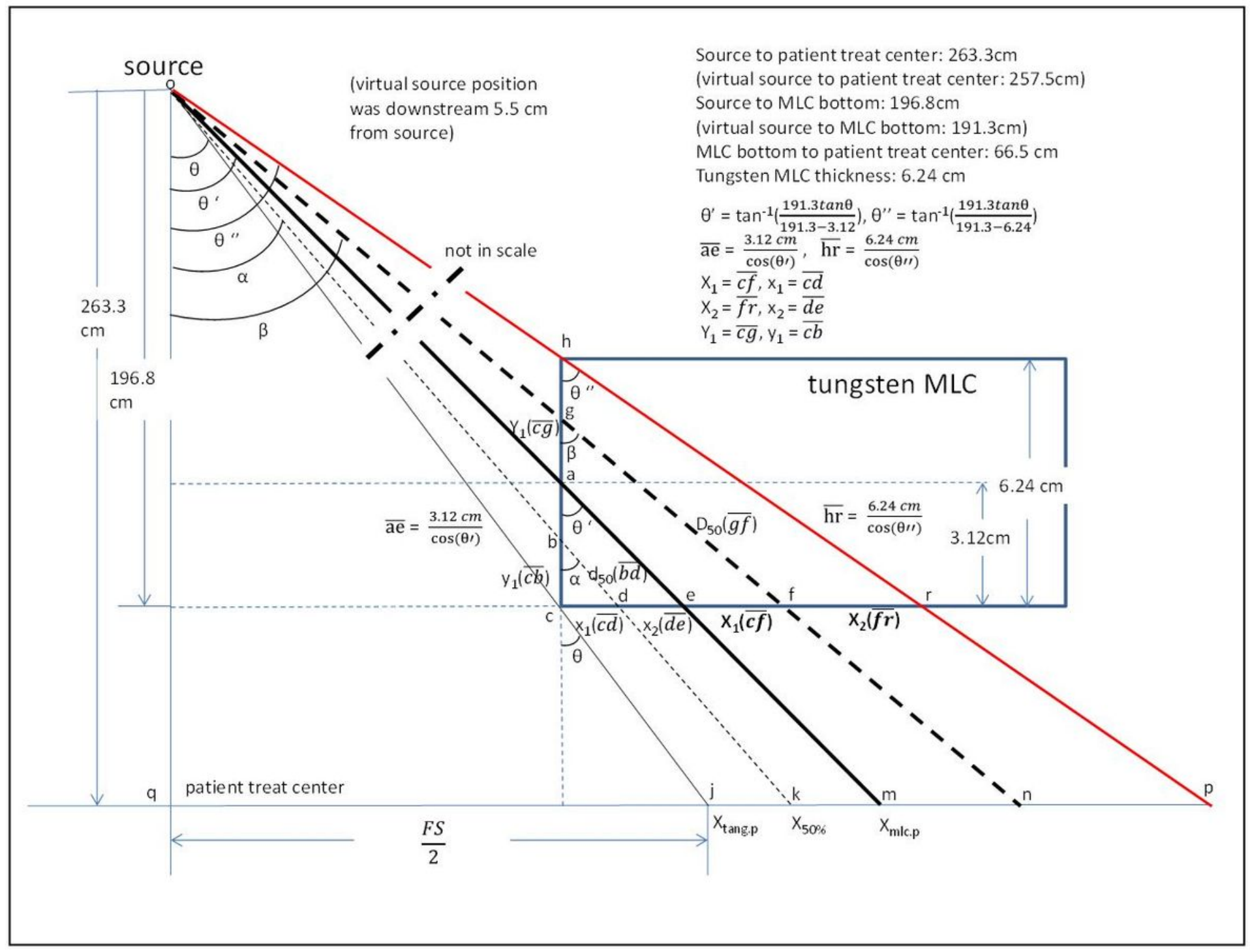

Figure 2

The definition of MLC nominal light field (visualized light field) edge, Xtang.p, the intersection of a line from the source to the leaf tip with an angle of $\theta^{\prime}$, Xmlc.p, and the dose $50 \%$ position $X 50 \%$ ( straggling range) of tungsten in treatment planning system. 


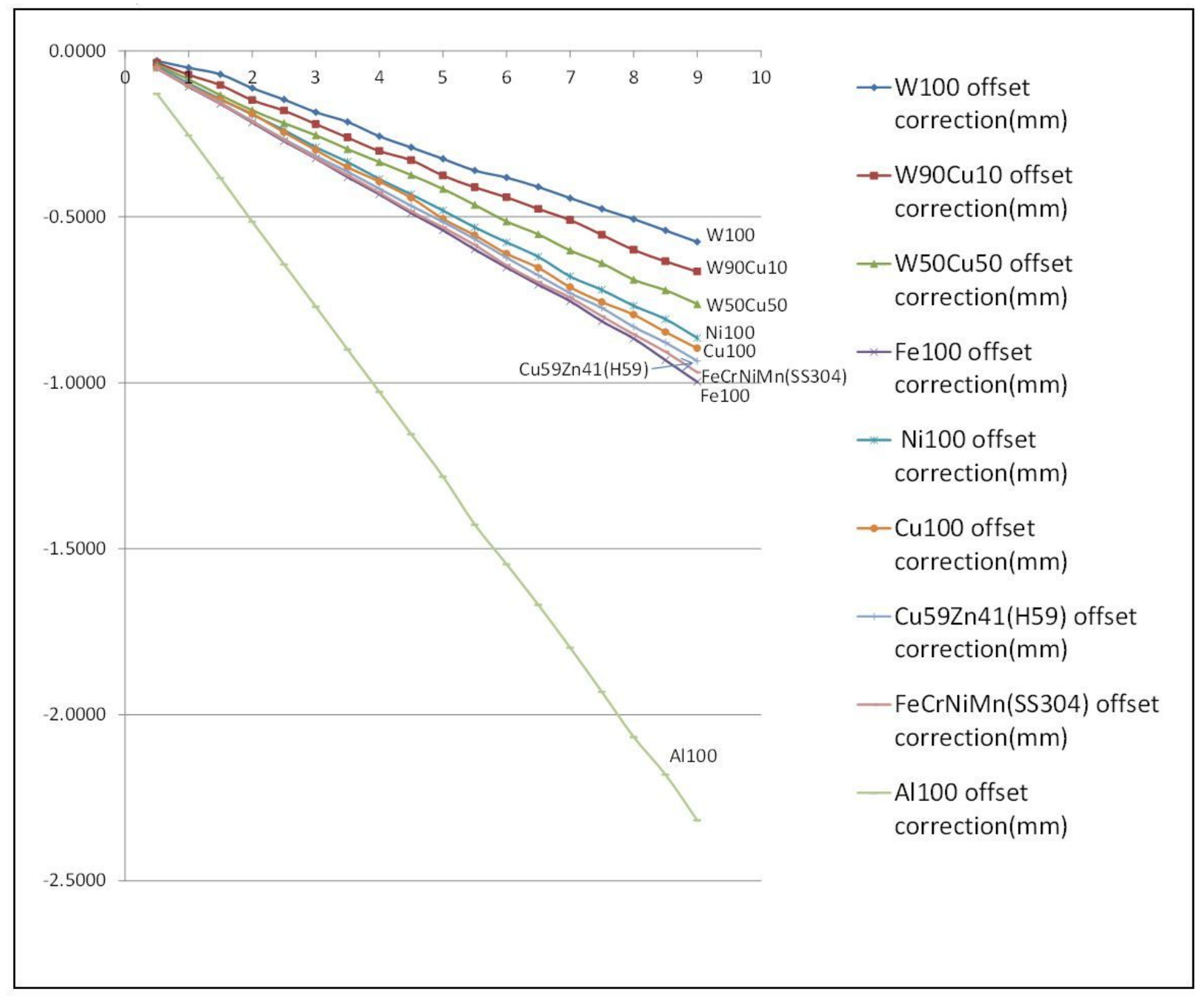

\section{Figure 3}

Schematic demonstrates the offset corrections of different material for MLC. The offset correction is increased because of the composition of different metal increased leading to the increasement of struggling ranges.

\section{Supplementary Files}

This is a list of supplementary files associated with this preprint. Click to download.

- AppendixAsupplement20210712.docx 